\title{
Time-spread echo digital audio watermarking tolerant of pitch shifting
}

\author{
Hironobu Takahashi*, Ryouichi Nishimura and Yôiti Suzuki \\ Research Institute of Electrical Communication, Tohoku University, \\ Katahira 2-1-1, Aoba-ku, Sendai, 980-8577 Japan
}

(Received 23 May 2005, Accepted for publication 25 July 2005)

Keywords: Digital audio watermarking, PN sequence, Time-spread echo method, Pitch frequency PACS number: 43.60.Dh [DOI: 10.1250/ast.26.530]

\section{Introduction}

As the number of Internet users increases, large amount of unauthorised digital data have been widely distributed through the Internet. Violation of author's copyright is thus a serious problem nowadays. Under these circumstances, digital watermarking is attracting attention as a promising technique to solve this problem. As for digital images, practical watermarking techniques have been already developed and used in DVD video systems [1]. Current digital audio watermarking techniques, in contrast, are immature in terms of its tolerance against malicious attacks. This is because the redundancy of audio signals is far less than that of image signals. Among several audio watermarking techniques developed in the past [2-4], a time-spread echo method showed good performances to certain attacks compared to the others [5]. However, pitchshifting, i.e., time stretch/compression was a very severe attack for the time-spread echo method. In this case, a conventional watermarking detection was completely ineffective. Therefore, we developed a new watermarking embedding/ detection method for the time-spread echo method to tackle with this problem. This method is based on adaptive modification of the time length of PN sequence corresponding to an instant pitch of music signal.

\section{Outline of the proposed time-spread echo method}

\subsection{Echo kernel}

In the time-spread echo method [5], watermarked signal is generated by convolving a time-spread echo kernel and an original audio signal. The echo kernel is composed of a unit impulse and a PN sequence as shown in Fig. 1. In Fig. 1, the parameter, $\alpha(0<\alpha \ll 1)$, is a factor to adjust the amplitude of PN sequence. Since the amplitude of the PN sequence is generally defined as 1 and -1 [6], it should be reduced by adjusting its amplitude so that listeners cannot detect the existence of the kernel. The parameter $\delta$ is the delay time between the unit impulse and the PN sequence. The parameter $L$ is the time length of the PN sequence.

To extract embedded information from a watermarked signal, the correlation of the PN sequence and the watermarked signal is used. The echo kernel and the delay time $\delta$ can be easily determined from the correlation.

*e-mail: h.takahashi@aist.go.jp

Current affiliation: National Metrology Institute of Japan/AIST
2.2. Encoding of information corresponding to pitch frequency

When a watermarked signal is exposed to a time stretch/ compression attack greater than $0.1 \%$, the conventional method completely fails to detect the watermarks. The reason is that the time length of the PN sequence embedded in the audio signal is changed. Hence, to be robust against this kind of attack, the PN sequence should be always kept the same length.

When an audio signal is exposed to a time stretch/ compression attack, pitch frequency of the audio signal is also changed. Hence, if the length of the PN sequence is related to the pitch frequency by a certain function, it can be estimated even when the watermarked signal is exposed to a time stretch/compression attack.

Figure 2 shows the block diagram of the proposed encoding process. First, to calculate the pitch frequency of the original audio signal, the original signal is divided into frames. Each frame is denoted by $x(N)$, where $N$ is frame number. Pitch frequency of the frame is denoted by $f_{x}$.

To estimate reliable pitch frequency, each frame is further divided into number of segments. Then, the most dominant frequency is adopted as the pitch frequency of the frame after summing up the obtained power spectral density of the segments in the target frame.

The time length of the PN sequence is adjusted by resampling the original PN sequence based on the obtained pitch frequency $f_{x}$. This part corresponds to "PN sequence adjuster" in Fig. 2. The original PN sequence is expressed as $P N_{\text {orig }}\left(L_{\text {orig }}\right)$, where $L_{\text {orig }}$ is the time length of the original PN sequence. The time length of a modified PN sequence, $L$, is expressed by

$$
L=\beta \cdot f_{x} \cdot L_{\text {orig }},
$$

where $\beta$ is the constant coefficient so as to adjust the time length of the modified PN sequence to a pre-determined value. The time length of the original PN sequence $L_{\text {orig }}$ is also constant. Hence, the time length of the modified PN sequence, $L$, varies only corresponding to the pitch frequency.

The modified PN sequence, $P N(L)$, is produced by resampling the original $\mathrm{PN}$ sequence, $P N_{\text {orig }}$, so that the time length of the modified PN sequence matches the length $L$. Then, the modified time-spread echo kernel is generated from the modified PN sequence and the unit impulse. The watermarked signal is generated by convolving this new echo kernel and the original audio signal. 


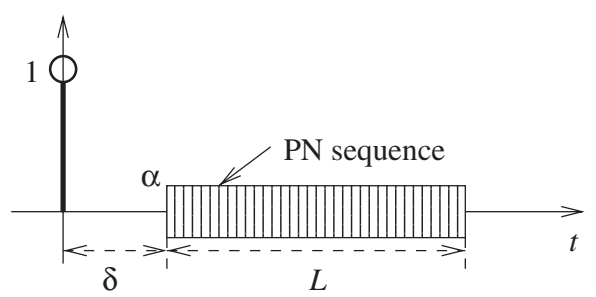

Fig. 1 Time pattern of the time-spread echo kernel.

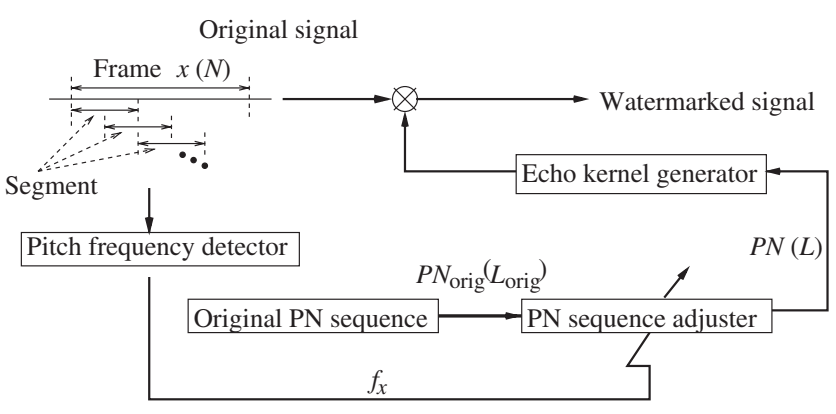

Fig. 2 Block diagram of encoding process.

2.3. Detection of embedded information in watermarked signal

To detect embedded information, detection of the pitch frequency is necessary because the PN sequence to be used in the decoding process is adjusted depending on the pitch frequency. The way to determine the pitch frequency and generate the PN sequence is the same as in the encoding process described in Section 2.2.

Figure 3 is a block diagram of the proposed decoding process. The frame of the watermarked signal $r(n)$ is given by

$$
r(n)=s(n) * k(n),
$$

where $s(n)$ is the frame of the original signal, $k(n)$ is the echo kernel and $[*]$ means a linear convolution. By applying cepstral analysis, $r(n)$ of Eq. (2) becomes

$$
c_{r}(n)=\operatorname{IFFT}(\log (\operatorname{FFT}(r(n)))),
$$

where $\operatorname{FFT}(\cdot)$ and $\operatorname{IFFT}(\cdot)$ mean Fourier transform and inverse Fourier transform, respectively. The decoded signal $d(n)$ is then obtained by the following equation:

$$
d(n)=\operatorname{xcorr}\left(c_{r}(n), P N\left(L^{\prime}\right)\right),
$$

where $\operatorname{xcorr}(\cdot)$ means cross-correlation, and $L^{\prime}$ is obtained by Eq. (2) using the estimated pitch frequency instead of $f_{x}$.

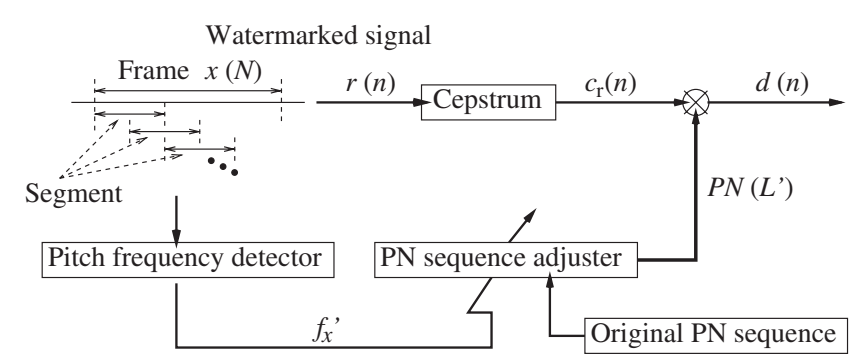

Fig. 3 Block diagram of decoding process.
Table 1 Music signals and the coefficient $\beta$ to be evaluated.

\begin{tabular}{cccc}
\hline & No. 1 & No. 2 & No. 3 \\
\hline genre & Pops & Classic & Piano \\
$\beta$ & 2.6 & 4.5 & 5.5 \\
\hline
\end{tabular}

\section{Evaluation of robustness against time stretch attack}

To evaluate the robustness of the proposed method, computer simulations were carried out using a few kinds of audio signals. Table 1 shows the audio signals to be examined. The signal No. 1 is a piece of pop music which contains vocals. The signal No. 2 is a piece of classical music played by an orchestra. The signal No. 3 is a piece of classical music with a piano. Sampling frequency was $44.1 \mathrm{kHz}$. The length of the signals was $60 \mathrm{~s}$. The signals were extracted not to include silent periods as much as possible, because the watermarking cannot work in silent periods theoretically. The length of the frame and segment were set to 11,045 points and 2,048 points, respectively. Each segment overlapped 1,024 points. Consequently, nine segments were used to determine the pitch frequency per one frame.

In the proposed method, the time length of the PN sequence in the echo kernel is proportional to the pitch frequency of a signal. Our previous research showed that the length of a PN sequence over 1024 points is efficient [5]. The coefficient $\beta$ in Eq. (1) was, therefore, set so that the time length of the PN sequence was 1024 points. The values of coefficient $\beta$ set in this evaluation are shown in Table 1. As for PN sequences, we selected M-sequence (Maximum-length sequence), because it was used in the conventional timespread echo method [5].

To evaluate the robustness, detection ratio $d$ was defined as follows:

$$
d[\%]=\frac{\text { correct frames }}{\text { total frames }} \times 100,
$$

where the term "correct frames" means the number of frames in which the embedded echo kernel was detected correctly, and the term "total frames" means the number of evaluated frames in the target audio signal.

Figure 4 shows the detection ratio of the proposed method when the watermarked signal was stretched from $-10 \%$ to $+10 \%$. For the sake of comparison, the result of the conventional method is also shown in Fig. 5. Figure 5 obviously shows that the detection ratio deteriorates drastically by a slight variation of the time stretching. In this case, the detection ratio becomes almost $0 \%$ as the time scale is stretched over $\pm 1 \%$. In contrast, from Fig. 4 , the detection ratio of the proposed method keeps over $55 \%$ even when the time scale is stretched from $-10 \%$ to $+10 \%$ regardless of the genre of music. The proposed method is, therefore, robust against the time stretch attack compared with the conventional method.

However, the proposed method has a problem. The conventional method could achieve almost $100 \%$ in case of no attack. In contrast, the detection ratio of the proposed method was around $85 \%$. Thus, we examined how correctly the pitch frequency was obtained from the watermarked signals without 


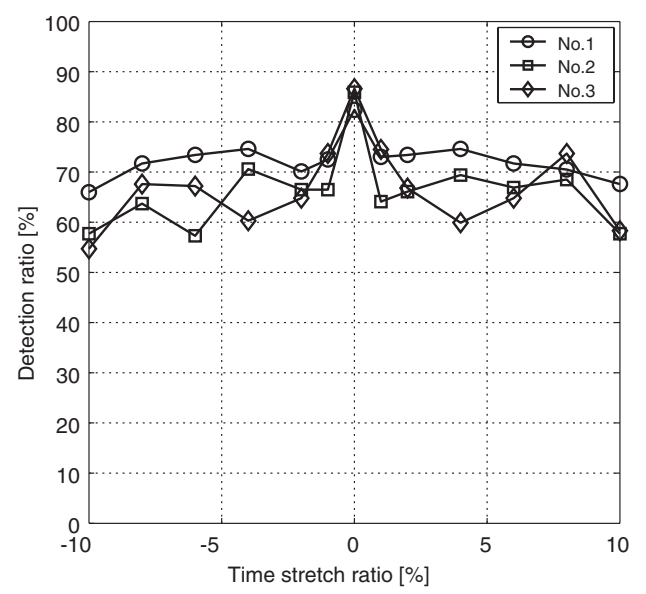

Fig. 4 The detection ratio, $d$, of the proposed method.

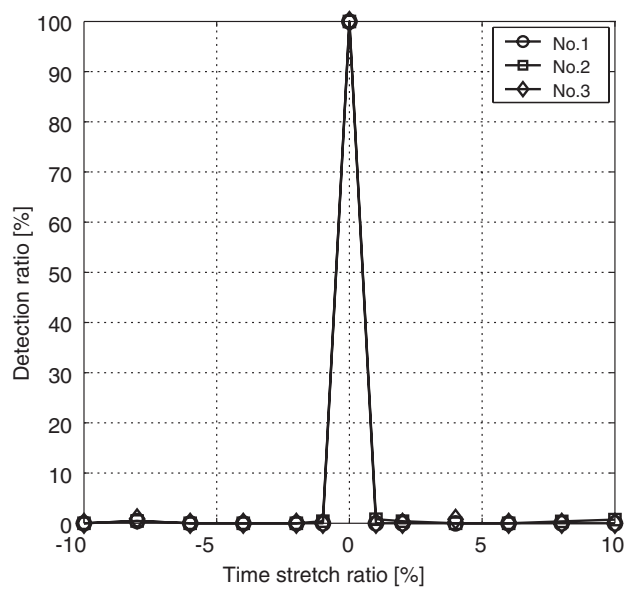

Fig. 5 The detection ratio, $d$, of the conventional method.

the time stretch attack. As a result, the ratio of correct pitch detection of the signals No. 1, No. 2 and No. 3 were $82.0 \%$, $84.5 \%$ and $94.7 \%$, respectively. These values are similar to the detection ratio shown in Fig. 4, though that of the signal No. 3 is somewhat less than the correct pitch detection ratio. Moreover, the proposed method failed to detect correct watermarks where the pitch frequency of the frame was missestimated. Hence, the performance of the proposed method depends on how correctly the pitch frequency can be detected.

For practical use, the detection ratio of watermark against all kinds of attacks should be as nearly $100 \%$ as possible. From this point of view, the proposed method as well as the conventional method have currently problems. The proposed method show still unsatisfactory detection rate of $65 \%$ on average against the time stretch/compression attacks though this value is substantially improved from that of the conventional. Moreover, while the conventional method achieved $100 \%$ detection in case of no attack, the proposed method exhibits poorer value. We consider this is mostly attributable to incorrect estimation of pitch frequencies. Thus, if the pitch frequency of the signal could be estimated correctly, the detection with the proposed method should be much improved, resulting that the inferior performance of the proposed method to that of the conventional one at no stretch/ compression case would also be markedly improved. Therefore, the proposed method is regarded as more promising than the conventional one though further investigation are still needed to make it more practical.

\section{Conclusion}

We propose a modified time-spread echo method in which the time length of PN sequence is varied corresponding to a pitch frequency. To evaluate the validity of the proposed method, computer simulations were carried out. Compared with the conventional time-spread echo method, the proposed method shows good robustness against a time stretch attack including pitch shifting.

\section{Acknowledgment}

A part of this study was supported by a Grant-in-Aid for Development of Innovative Technologies (Millennium Project, 12107) by the ministry of Education, Culture, Sports, Science and Technology, Japan.

\section{References}

[1] G. C. Langelaar, I. Setyawan and R. L. Lagendijk, "Watermarking digital image and video data. A state-of-the-art overview," IEEE Signal Process. Mag., 17, 20-46 (2000).

[2] L. Boney, A. H. Tewfik and K. N. Hamdy, "Digital Watermarks for Audio Signals," Proc. EUSIPCO-96, Vol. 3, pp. 1697-1700 (1996).

[3] R. Nishimura, M. Suzuki and Y. Suzuki, "Proposal of a watermarking for music sound using a time-variant all-pass filter," Tech. Rep. IEICE, EA2001-36, pp. 65-72 (2001).

[4] W. Bender, D. Gruhl and N. Morimoto, "Techniques for data hiding," Proc. SPIE, pp. 164-173 (1995).

[5] B.-S. Ko, R. Nishimura and Y. Suzuki, "Time-spread echo method for digital audio watermarking using PN sequences," Proc. ICASSP-2002, pp. 387-392 (2002).

[6] J. G. Proakis, Digital Communications (McGraw-Hill, New York, 1995). 